\title{
Retrospective study of maxilla growth in a Spanish population sample
}

\author{
Juan Alió-Sanz ${ }^{1}$, Carmen Iglesias-Conde ${ }^{2}$, José-Lorenzo Pernía ${ }^{3}$, Alejandro Iglesias-Linares ${ }^{4}$, Asunción \\ Mendoza-Mendoza ${ }^{5}$, Enrique Solano-Reina ${ }^{6}$
}

${ }^{1}$ DDS, MS, PhD. Professor of orthodontics, Universidad Complutense de Madrid, Spain

${ }^{2} \mathrm{DDS}, \mathrm{PhD}$. Private practice, Orense, Spain

${ }^{3}$ DDS , PhD. Associate professor, Department of orthodontics, Universidad Complutense de Madrid, Spain

${ }^{4}$ DDS , MSc, PhD. Lecturer Masters Programme in Orthodontics and Dentofacial Orthopaedics. School of

Dentistry. University of Seville

${ }^{5} \mathrm{DDS}, \mathrm{MSc}$, PhD. Professor of paedriatic dentistry, University of Seville. Spain

${ }^{6}$ DDS , MSc, PhD. Chairman of orthodontics, University of Seville. Spain

Correspondence:

Complutense University of Madrid, Spain.

Herreros de Tejada, 24

28016 Madrid (Spain)

jalio@iberortodoncia.com

Received: 24/04/2010

Accepted: 11/07/2010

Alió-Sanz J, Iglesias-Conde C, Pernía JL, Iglesias-Linares A, MendozaMendoza A, Solano-Reina E. Retrospective study of maxilla growth in a Spanish population sample. Med Oral Patol Oral Cir Bucal. 2011 Mar 1;16 (2):e271-7.

http://www.medicinaoral.com/medoralfree01/v16i2/medoralv16i2p271.pdf

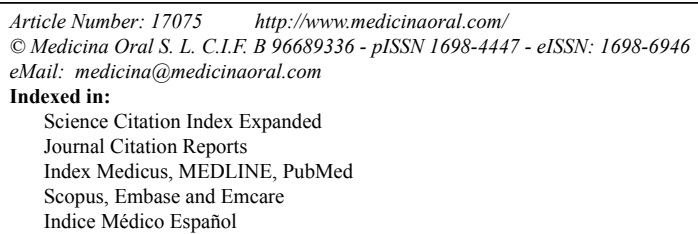

\begin{abstract}
This study has been designed to evaluate the vertical and sagittal changes in the maxilla due to growth. A sample group was chosen of 38 individuals with normal occlusion, composed of 16 females and 22 males between the ages of 8 and 18. The total sample was divided into three groups: prepubescent (8-11 years), pubescent (12-14 years) and post-pubescent (15-18 years). A series of cephalometric angle parameters (SNA, maxillary height, slope of the palatal plane and maxillary depth) and lineal parameters (effective maxillary length, palatal plane length, middle third of the face height and convexity) were traced. Superimpositions of the initial and final cephalometries in the $\mathrm{Ba}-\mathrm{N}$ plane and in the Nasion fixed point were carried out to measure growth. An analytic statistical analysis was applied using a Student $t$ test for independent samples in order to evaluate the differences found according to sex. An analysis of variance followed by Duncan's multiple range test was done to study the evolution of each variable throughout the duration of the experiment. In light of the results obtained, we have come to the following conclusions: sagittal growth of the maxilla is constant from the age of 8 to 18 years with an average increase of $0.2 \mathrm{~mm} /$ year. Vertical growth, as well as general maxillary growth, is greater in the prepubescent group.
\end{abstract}

Key words: Orthodontics, craniofacial growth, maxilla growth, cephalometry, superimposition. 


\section{Introduction}

The study and evaluation of general craniofacial growth has been going on in orthodontics for a very long time. (1-3) This is because growth can favorably or unfavorably affect treatment. Some systems try to predict growth to plan treatment accordingly (4), even though some studies have questioned the effectiveness of these predictions. (5) When evaluating growth, it is crucial to know how it is occurring - in what direction and how much more growth remains - at the time orthodontic treatment begins. In general, it is agreed that growth of the different craniofacial skeletal components does not happen at the same rate nor does it follow a common pattern. However, some parameters can remain constant, such as the ratio between the upper depth of the face and its width.

Numerous studies have looked into the changes one's face undergoes from childhood to adulthood. Nanda and Ghosh (6) carried out a longitudinal study on the Denver Child Research Council finding that maximum growth occurs between the ages of 6 and 12 years in girls and between 12 and 18 years in males, and that between the ages of 18 and 24 there is some residual growth in both sexes. Some authors have studied craniofacial skeletal changes in subjects older than 18 . For instance, Bishara (7) found that upper and posterior facial height lessened in women starting at fifteen years old. The SNB angle and the SN-Pog increased in males between the ages of 15 and 25 , while the facial height quotient fell in this group. West et al. (8) studied the changes that can be found in some cephalometric variables between the ages of 17 and 48 . He found that total mandibular length, posterior facial height and anterior facial height $(\mathrm{N}-\mathrm{Me})$ increased during this long period of observation.

The normal procedure for studying and analyzing growth over a long period has been cephalometry. This method, introduced in the 1930s (9), has allowed specialists to study craniofacial skeletal changes over time "in vivo" by superimposing cephalometries. Brodie1 pointed out the concentric growth of calvarial bones and Bjork (2) came up with the concept that the rotations involved in growth are normal characteristics of human facial development. Lande (3) had already discussed how the slope of the mandibular ridge tends to become less inclined with growth. One variable which must be borne in mind in growth studies is skeletal pattern and facial type. Brodiel affirmed that the morphogenetic growth pattern is established quite early on and that it varies very little.

With respect to growth of the nasomaxillary complex, Bjork (10) noted that the Nasion point moves forward throughout the whole growing period, but it can also move up or down. Something similar happens with the sella turcica point, which moves towards the back during growth, but it can also move up or down. Enlowl1 studies postnatal maxillary growth in detail noting that it is similar to that of the mandible, i.e., forward and down. This growth is especially evident at the back, thereby easing the adaptation to permanent dentition. According to this same author, the palatal process normally grows downwards due to the combination of bone apposition in the oral palatal surface and re-absorption in the nasal area. The pre-maxillary area moves downward.

At first, many studies1 (12), stated that vertical growth of the maxilla was only downwards, without any rotational movement at all. Later studies $(10,13)$ showed that the descent and forward movement of this bone occurs at the same time as slight rotational changes.

All of the changes that take place in the maxilla during growth can be seen through different cephalometric analyses. Few authors have exclusively studied maxillary behavior (14). However, the cephalometric changes that occur in this bone as a consequence of growth are very important when planning orthopedic treatment in Class II patients or those with biprotrusion or a vertical syndrome. Therefore, this study evaluated the changes that occur in some cephalometric parameters that measure sagittal and vertical maxillary position in a sample of normally occlusive patients in Spain.

\section{Materials and Methods}

\section{-Subjects}

The sample used in this study is made up of 38 individuals, 16 females and 22 males, ranging in age from 8 to 18 years. The average age at the beginning of the study was 10 years $/ 4$ months for females and 10 years $/ 1$ month for males. At the end of the study, the average age for females was 16 years $/ 6$ months and for males, 16 years/ 7 months. The total sample group was divided into three groups based on age: prepubescent (8 to 11 years old), pubescent (12 to 14 years old) and post-pubescent (15 to 18 years old).

\section{-Selection criteria}

The selection criteria for the sample were the following: a) the subject must have been between the ages of 8 and 18 at the first visit; b) an absence of apparent craniofacial deformities and occlusal stability; c) not have had craniofacial traumatisms in the past; d) not have any congenital anomalies; e) in the extraoral examination selected patients must present an adequate balance among the three thirds of the face; and f) intraorally clear Class I molar and canine intercuspidation; g) in addition, the subject must not have undergone the extraction of any permanent teeth, have had any changes made to the size, number or shape of any teeth nor show any negative bone-dental discrepancies in either arch; h) subjects must not have undergone orthodontic treatment or oral surgery nor sought orthodontic treatment prior to or throughout the duration of the study; i) sub- 
jects must be from a Caucasian origin.

-Radiographic and cephalometric records

A lateral cranial x-ray was taken of each subject yearly throughout their participation in the study as an annual general revision protocol stated in the Complutense School of Dentistry of Madrid. A cephalometry was traced for all the lateral cranial x-rays using computer analysis (Nemoceph Studio, Nemotec Dental System) marking the following cephalometric landmarks: Sella turcica (S), Basion (Ba), Nasion (N), point A, Anterior nasal spine (ANS), Posterior nasal spine (PNS), Porion (Po), Pterygomaxillary suture (Cf), suborbital point (Or), Condylion point (Co) and Pogonion (Pg). (Fig. 1). Cephalometric parameters were traced over the following angular and lineal landmarks, respectively SNA (S$\mathrm{N}-\mathrm{A}$ angle), maxillary height (EN A-Cf-N angle), slope of the palatal plane (palatal plane-Frankfort angle) and maxillary depth (N-A Frankfort) and lineal effective maxillary length (Condylion to point $\mathrm{A}$ ), palatal plane length (EN A-ENP distance), middle third facial height (N-ENA distance) and convexity (Point A to facial plane).

In order to evaluate growth, we superimposed the initial tracings over the final tracings in the Ba-N plane with $\mathrm{N}$ as a fixed point (4). The initial and final point $\mathrm{A}$ were projected over the Frankfort plane as a horizontal reference. We used the pterygoid as a vertical reference projecting over it both the first and last registers of the anterior and posterior nasal spine. Positive values were applied when the final point A was more forward than the initial point A. Likewise, a positive value was applied when the final nasal spine positions were below the initial positions. Any rotations in the palatal plane were also taken into consideration. A positive rotation was defined as when the final palatal plane position had undergone a counterclockwise change with respect to the initial position. Otherwise, it was given a negative value. (Fig. 2)

-Statistics

All the cephalometries were traced by two experimented researchers (J.A.S. and C.I.C.) belonging to the general research project on growth carried out in the Master's Program in Orthodontics at the Universidad Complutense of Madrid. These researchers calibrate their measurements annually to avoid any error in the cephalometric tracings. In order to estimate the intraexaminer variation for the radiological evaluation all the radiographs were evaluated twice by the same experienced examiner (J.A.S.). In order to estimate the interexaminer variation all the radiographs were evaluated by a second experienced examiner (C.I.C.).

Once both researchers have performed the tracings, they are compared to each other thereby obtaining one of three distinct possibilities:

1) Type I Concordance: total coincidence of the trac-

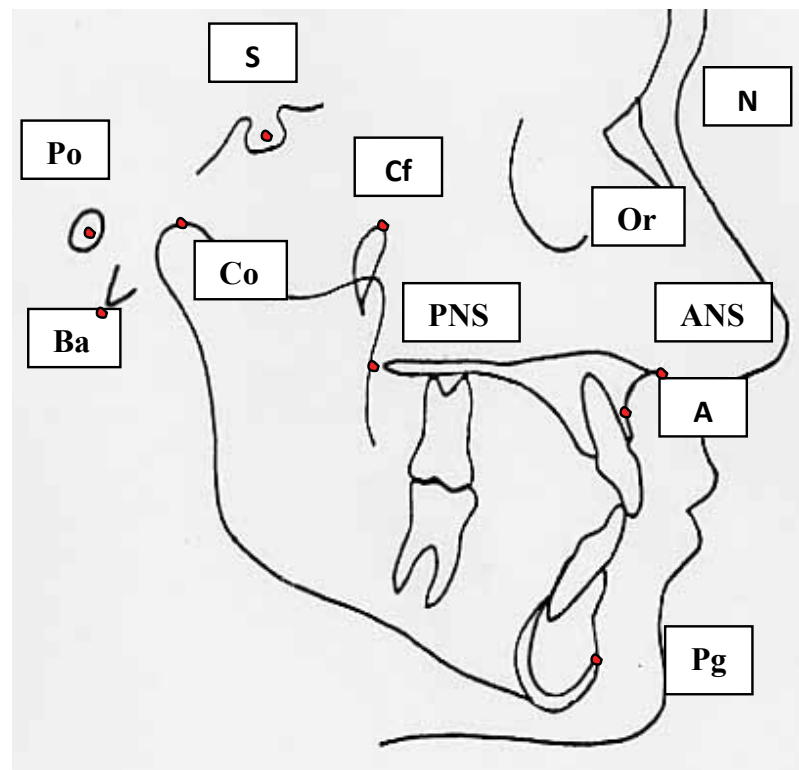

Fig. 1. Cephalometric landmarks used in the study. Sella turcica (S), Basion (Ba), Nasion (N), point A (A), Anterior nasal spine (ANS), Posterior nasal spine (PNS), Porion (Po), Pterygomaxillary suture (Cf), suborbital point (Or), Condylion point (Co) and Pogonion (Pg).

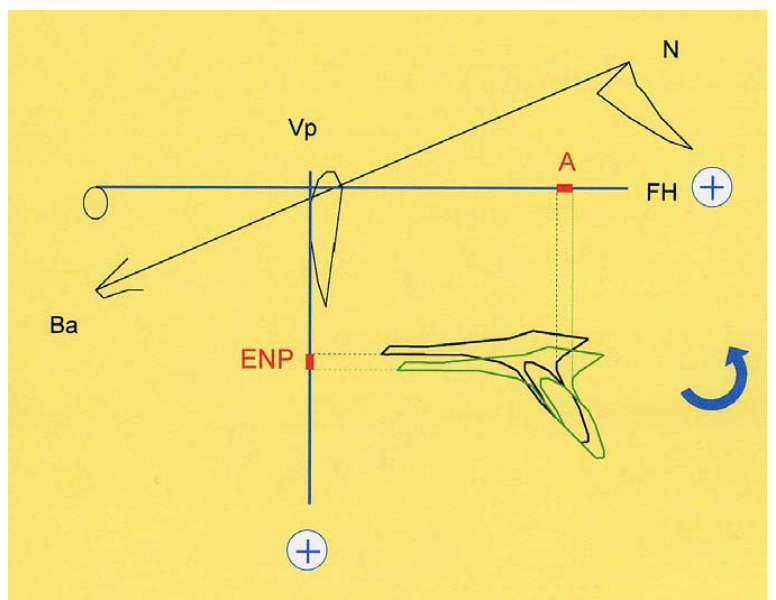

Fig. 2. Superimposition of the maxilla. Ba-N in the N plane. Nasion $(\mathrm{N})$; vertical point (Vp); Basion $(\mathrm{Ba})$; Frankfort plane $(\mathrm{FH})$; posterior nasal spine(PNS); point A (A).

ings.

2) Type II Concordance: difference in some parameter among the tracings that are less than the following values: Convexity: Less than $1 \mathrm{~mm}$; Maxillary height: Less than $3^{\circ}$;

Effective maxillary length: Less than 3mm; Palatal plane length: Less than 2mm; SNA: Less than $3^{\circ}$; Maxillary depth: Less than $3^{\circ}$; Slope of the palatal plane: Less than $1^{\text {o }}$; Middle third of face height: Less than $2 \mathrm{~mm}$.

3) Type III Concordance: Greater difference than de- 
scribed above.

In Type II concordance the arithmetic mean is established between the two parameter values that do not coincide. When the difference is greater (Type III concordance) the tracings are done again and are referenced against the three concordance possibilities mentioned above.

The causal error was determined using Dahlberg's formula36 (S.E. $=\sqrt{ }^{-} \mathrm{d}^{2} / 2 \mathrm{n}$ ) and the systematic error using a $\mathrm{t}$ test for a $\mathrm{P}<0.05$.

A descriptive statistical analysis was used to evaluate the data obtained in which the arithmetic mean, standard deviation, percentiles and rank (maximum and minimum values) of each variable by sex and age group were included. Later, an analytical or inferential statistical analysis was done. In order to study the evolution of each variable over time and establish comparisons in the behavior shown by any one variable in each age group, an analysis of variance (ANOVA) followed by Duncan's multiple range test was used as a test a posteriori with a reliability of 0.05 . A Student $t$ for independent samples was applied to study the differences in function of sex.

\section{Results}

Regarding the effective maxillary length parameter observed for the different groups, a logical progressively higher value was registered for the older groups, with statistical significance. On the other hand, the palatal plane length parameter differed significantly between the prepubescent group and both of the others, which did not show those differences. Thus, a substantial change in palatal plane length can be inferred during the ages of 8-11 years compared to the remaining growth period until adulthood (Table 1).

Regarding gender-relative differences (Table 1) the Maxilla height and palatal plane angle with SN parameters were found to be higher in girls compared to those obtained for boys. While angular measurements were found to be higher in girls, linear parameters, maxillary length and palatal plane length were greater in males. (Table 1)

Regarding initial and final superimposition values (Table 2), an advancement of point $\mathrm{A}$ has been noted in all final values of the groups studied. This advance of point A was less in the oldest age group even though significant differences did not appear among the three groups.

The anterior nasal spine (ANS) always moves down in all the age groups by approximately $1 \mathrm{~mm}$, but significant differences did not appear. Similar behavior was seen in the posterior nasal spine even though its downward movement is somewhat greater if compared to that of the anterior nasal spine. A statistically significant difference between the post-pubescent group and the other two was found in that there is less downward movement of the posterior spine in prepubescent and pubescent groups. Due to the slightly different downward movement of the two nasal spines, the palatal plane tends to have a positive slope in the first two age groups, while turning in the opposite direction in the post-pubescent group. (Table 2)

The differences according to sex are reflected in the four cephalometric superimposition variables (Table 2). As can be seen, the advance of the point $\mathrm{A}$ is greater in females, with a statistical significance between each

Table 1. Maxillary cephalometric variables according to age and gender groups

\begin{tabular}{|l|c|c|c|c|c|c|}
\cline { 2 - 6 } \multicolumn{2}{l|}{} & \multicolumn{3}{c|}{ Age groups } & \multicolumn{2}{c|}{ Gender groups } \\
\hline Variable & Total & Pre-pubescent & Pubescent & Post-pubescent & Girls & Boys \\
\hline SNA & $79,27 \pm 3,19$ & $78,74 \pm 2,67$ & $79,72 \pm 3,47$ & $79,68 \pm 3,86$ & $78,44 \pm 3,53$ & $79,92 \pm 2,75$ \\
\hline Maxillary height & $58,88 \pm 3,01$ & $58,41 \pm 3,01$ & $59,13 \pm 2,95$ & $59,85 \pm 3,02$ & $59,40 \pm 2,81$ & $* 58,44 \pm 3,09$ \\
\hline Maxillary depth & $90,55 \pm 2,69$ & $90,19 \pm 0,69$ & $90,92 \pm 2,65$ & $90,94 \pm 2,78$ & $90,69 \pm 3,05$ & $90,44 \pm 2,37$ \\
\hline PP-SN & $5,42 \pm 3,39$ & $5,16 \pm 3,09$ & $5,70 \pm 3,71$ & $5,34 \pm 3,43$ & $6,82 \pm 3,02$ & $* * 4,31 \pm 3,27$ \\
\hline Convexity & $0,54 \pm 2,81$ & $0,20 \pm 2,74$ & $0,88 \pm 2,83$ & $0,62 \pm 3,03$ & $0,77 \pm 3,18$ & $0,36 \pm 2,48$ \\
\hline $\begin{array}{l}\text { Effective length of } \\
\text { maxilla }\end{array}$ & $88,57 \pm 4,65$ & $* 86,30 \pm 4,22$ & $* 90,02 \pm 4,16$ & $* 92,15 \pm 3,54$ & $87,06 \pm 4,34$ & $* * 89,76 \pm 4,56$ \\
\hline Palatal plane length & $53,23 \pm 3,46$ & $* 51,48 \pm 2,88$ & $* 54,42 \pm 3,15$ & $* 55,71 \pm 3,42$ & $52,53 \pm 2,66$ & $* 58,44 \pm 3,09$ \\
\hline Ant.-sup. Facial height & $54,3 \pm 3,53$ & $* 52,52 \pm 3,33$ & $* 55,50 \pm 3,04$ & $* 56,86 \pm 2,52$ & $54,69 \pm 3,41$ & $53,98 \pm 3,61$ \\
\hline
\end{tabular}

SNA: Sella-Nasion plane to Nasion-Point A angle; PP-SN: Palatal plane to Sella-Nasion planeangle; ${ }^{*} \mathrm{p}<0.05 ;{ }^{* *} \mathrm{p}<0.01$. 
Table 2. Asseessment of maxillary growth through cephalometric superimposition analysis accordingto age and gender groups.

\begin{tabular}{|l|l|c|c|c|c|c|}
\cline { 2 - 7 } \multicolumn{1}{l|}{} & \multicolumn{3}{c|}{ Age groups } & \multicolumn{2}{c|}{ Gender groups } \\
\hline Variable (mm/year) & Total & Pre-pubescent & Pubescent & Post-pubescent & Girls & Boys \\
\hline Ai - Af & $0,20 \pm 0,29$ & $0,22 \pm 0,31$ & $0,19 \pm 0,28$ & $0,11 \pm 0,21$ & $* * 0,29 \pm 0,37$ & $* * 0,13 \pm 0,18$ \\
\hline ENAi-ENAf & $0,98 \pm 0,65$ & $1,05 \pm 0,47$ & $0,97 \pm 0,85$ & $0,48 \pm 0,25$ & $* * * 0,71 \pm 0,47$ & $* * * 1,21 \pm 0,69$ \\
\hline ENPi-ENPf & & & & & & \\
\hline PPi-PPf & $1,02 \pm 0,58$ & $1,09 \pm 0,49$ & $1,03 \pm 0,68$ & $* 0,49 \pm 0,27$ & $* * 0,84 \pm 0,61$ & $* * 1,18 \pm 0,51$ \\
& $0,08 \pm 0,68$ & & & & $0,07 \pm 0,69$ & $0,08 \pm 0,67$ \\
\hline
\end{tabular}

Ai- Af: poimt A initial position minus point A final position; ENAi- ENAf: Initial anterior nasal spine position minus final anterior nasal spine position; ENPi- ENPf: Initial posterior nasal spine position minus final position nasal spine positon; PPi-PPf: Initial palatal plane position minus final palatal plane position; *: $<<0,05 ; * *: p<0,01 ; * *: p<0,001$.

value $(\mathrm{P}<0.01)$. However, the ANS moves down more in males, thereby revealing statistical significance between each group in this case as well. The same happens with the PNS, which shows the same statistical difference. The changes observed in the slope of the palatal plane are practically equal in both sexes. (Table 2)

\section{Discussion}

-Sagittal growth of the maxilla

Sagittal growth of the maxilla has been studied using SNA angular measurements and maxillary depth and convexity as lineal measurements. The SNA angle increased slightly from the youngest to the oldest group. However, there were not significant differences among the three groups. These results support those obtained by other authors 14 as far as the maxilla show growth similar to that of the anterior cranial base in the sagittal plane. However, some differences are found in the value of this angle. Ochoa et al. (15), on the other hand, do not find differences in this measurement in the different age groups. Nonetheless, we do find differences in the total value of the angle, since the total average was $79.27^{\circ}$ in our sample, while Ochoa et al. came up with an average of $81.44^{\circ}$. Our values were also lower than those recorded by Rothstein et al. (16) in their longitudinal study on Class II Division I malocclusion. This difference could be due to the different ethnic make-up of each sample group. Maxillary depth also underwent a slight increase between the youngest and oldest groups. As in the case of the SNA, the differences were not significant. We did not find significant differences in the sexes with respect to these measurements. Braun (17) did not find diffe- rences between the sexes until the subjects reached 14 years of age, after that these authors found that sagittal growth tended to be less in females and stopped completely at the age of 16 .

In order to measure sagittal growth of the maxilla independently of the anterior cranial base, we used cephalometric superimpositions measuring growth with the projection of point A over the Frankfort plane (Table 2). Maximum growth occurred in the prepubescent group although there were not significant differences among the three age groups. Therefore, one can conclude that the antero-posterior growth of the bone is quite constant between the ages of 8 and 18 years. The average growth of point A was $0.20 \mathrm{~mm} /$ year. Pollard et al. (18) found a significant maxillo-mandibular growth rate in males in late developmental stages. We also obtained these results since growth in the post-pubescent group, even though less than in the other two groups, did not show significant differences, nor did they appear between the two sexes.

-Vertical maxillary growth

We have studied vertical growth of the maxilla with reference to maxillary height, middle facial third height and the result of the initial and final superimpositions of the anterior and posterior nasal spine. An increase in maxillary height is observed though there are not significant differences among the three groups. However, middle facial third height is different in the prepubescent group when compared to the other two. Balanced growth of the structures that make up the angle of the middle third of the face prevent the angular value from showing significant differences. However, greater verti- 
cal growth of the maxilla during the prepubescent stage does affect the lineal value of middle facial third height. This value is quite stable throughout growth. In a later study, Karlsen (19) points out that an increased middle facial height could indicate a tendency to a maxillary protrusion.

When we analyzed the superimpositions we observed that the average growth of the anterior nasal spine was $0.98 \mathrm{~mm} /$ year with no differences among the three groups. However, the posterior nasal spine moves down more, except in the post-pubescent group, in which the downward movement is less. The greater posterior growth makes the slope of the palatal plane increase, especially in the prepubescent and pubescent groups. Classic studies by Brodiel and Bjork (2) admit that there is basically parallel vertical maxillary growth. However, Bjork (10) does allow the possibility for a clockwise or counterclockwise rotation of this bone during its vertical growth. Whether it rotates in one direction or the other will primarily depend on the re-absorptive process of the nasal fossa floor being anterior or posterior. Other studies find that the changes in slope of this plane are minimal.14

It is important to underline the fact that there are differences between the sexes in that the anterior and posterior downward movement of the maxilla is greater in males than in females (Table 2). Ferrario et al.20 found differences in the values of facial heights between the two sexes, but they also found them between females in the prepubescent and pubescent groups. We found differences between sexes in maxillary height but not in middle facial height. This result, although it reached a low level of statistical significance $(p<0.05)$, is at least questionable since the greatest differences between sexes usually appear in lineal rather than in angular measurements. Finding differences between the sexes is common in many growth studies.7

-Size of the maxilla.

We have analyzed maxillary size in this study using two variables: effective maxillary length and palatal plane length. The results of these two variables have been studied in relation to the changes in the cephalometric superimpositions between the initial point A and the final one. Effective maxillary length increased significantly $(\mathrm{P}<0.05)$ in all the age groups. The biggest differences were found between the prepubescent and pubescent groups and were significantly greater in males. Ochoa15 found that maxillary length increased in all the groups and then stabilized starting at 14 years of age. In our results there is a significant increase in this variable after this age, just as was found in males by Nanda and Ghosh.5 Our results agree with those of these authors in that the greatest increase of this variable occurs in the prepubescent group. Palatal plane length also increased in all the groups but was only significant in the prepubescent group with respect to the other two. This can be interpreted to mean that various structures intervene in sagittal growth of the maxilla. One of these structures, the palatal plane, is responsible for growth in the prepubescent stage. In the other two stages other adjacent structures directly intervene, such as the palatal bone or movement caused by the growth of the cranial base. In addition, the superimpositions of the initial and final point A not revealing significant differences in the three age groups would suggest that the growth is fundamentally posterior. Nonetheless, the difference found between our measurements and Karlsen's19 with reference to effective maxillary length and palatal plane length is surprising, given that ours are much greater. This would seem to suggest that caution must be taken when interpreting comparisons of cephalometric standards, especially lineal standards, among subjects of different ethnic origins.

\section{Conclusions}

1. Sagittal growth of the maxilla was quite constant from 8 to 18 years of age, averaging $0.20 \mathrm{~mm} /$ year. This growth was significantly greater in females.

2 . The greatest vertical growth of the maxilla was seen in the prepubescent group (8-11 years old). However, greater growth of the posterior nasal spine in relation to the anterior takes place in the pubescent and postpubescent groups.

3. For the same age period studied, vertical growth of the maxilla was significantly greater in males, while sagittal maxillary growth, measured from point A, was greater in females.

\section{References}

References with links to Crossref - DOI

1. Brodie AG. On the growth pattern of the human head from the third month to the eighth year of life. Am J Anat. 1941;68:209-262.

2. Bjork A. Facial growth in man, studied with the aid of metallic implants. Acta Odontol Scand. 195;13:9-34.

3. Lande C. Growth behaviour of human bony facial profiles revealed by serial cephalometric roentgenology. Angle Orthod 1952;22:7890.

4. Ricketts RM. A four-step method to distinguish orthodontic changes from natural growth. J Clin Orthod. 1975;9:208-15, 218-28.

5. Nanda RS, Ghosh J. Longitudinal growth changes in the sagittal relationship of maxilla and mandible. Am J Orthod Dentofacial Orthop. 1995;107:79-90.

6. Nanda SK. Patterns of vertical growth in the face. Am J Orthod Dentofacial Orthop. 1988;93:103-16.

7. Bishara SE, Peterson LC, Bishara EC. Changes in facial dimensions and relationships between the ages of 5 and 25 years. Am J Orthod. 1984;85:238-52.

8. West KS, McNamara JA Jr. Changes in the craniofacial complex from adolescence to midadulthood: a cephalometric study. Am J Orthod Dentofacial Orthop. 1999;115:521-32.

9. Broadbent BH. A new X-ray technique and its application to orthodontia. Angle Orthod 1931;1:45-66.

10. Björk A, Skieller V. Growth of the maxilla in three dimensions as revealed radiographically by the implant method. Br J Orthod. 1977;4:53-64.

11. Enlow DH, Bang S. Growth and remodeling of the human maxilla. Am J Orthod. 1965;51:446-64. 
12. Bjork A. Cranial base development. A follow up X ray study of the individual variation in growth occurring between the ages 12 and 20 years and its relation to brain case and face development. Am J Orthod 1955;41:198-225.

13. Björk A, Skieller V. Normal and abnormal growth of the mandible. A synthesis of longitudinal cephalometric implant studies over a period of 25 years. Eur J Orthod. 1983;5:1-46.

14. Nanda RS, Merrill RM. Cephalometric assessment of sagittal relationship between maxilla and mandible. Am J Orthod Dentofacial Orthop. 1994;105:328-44.

15. Ochoa BK, Nanda RS. Comparison of maxillary and mandibular growth. Am J Orthod Dentofacial Orthop. 2004;125:148-59.

16. Rothstein T, Yoon-Tarlie C. Dental and facial skeletal characteristics and growth of males and females with class II, division 1 malocclusion between the ages of 10 and 14 (revisited)-part I: characteristics of size, form, and position. Am J Orthod Dentofacial Orthop. 2000;117:320-32.

17. Braun S, Rudman RT, Murdoch HJ, Hicken S, Kittleson R, Ferguson DJ. C-axis: a growth vector for the maxilla. Angle Orthod. 1999;69:539-42.

18. Pollard LE, Mamandras AH. Male postpubertal facial growth in Class II malocclusions. Am J Orthod Dentofacial Orthop. 1995;108:62-8.

19. Karlsen AT. Association between facial height development and mandibular growth rotation in low and high MP-SN angle faces: a longitudinal study. Angle Orthod. 1997;67:103-10.

20. Ferrario VF, Sforza C, Poggio CE, Colombo A, Cova M. Effect of growth and development on cephalometric shapes in orthodontic patients: a Fourier analysis. Eur J Orthod. 1997;19:669-80.

\section{Acknowledgments}

This work has been financed by PAI CTS-353 research group from the Consejería de Innovación Ciencia y Empresa of the Government of Spain. 